\title{
Smooth printing of viscoelastic microfilms with a flow focusing ejector
}

\author{
A. Ponce-Torres ${ }^{\mathrm{a}}$, E. J. Vega ${ }^{\mathrm{a}, *}$, A. A. Castrejón-Pita ${ }^{\mathrm{b}}$, J. M. Montanero ${ }^{\mathrm{a}}$ \\ ${ }^{a}$ Depto. de Ingeniería Mecánica, Energética y de los Materiales and \\ Instituto de Computación Cientíica Avanzada (ICCAEx), \\ Universidad de Extremadura, E-06006 Badajoz, Spain \\ ${ }^{b}$ Department of Engineering Science, University of Oxford, Parks Road, Oxford OX1 \\ 3PJ, United Kingdom
}

\begin{abstract}
The flow focusing principle has recently been shown to successfully generate micrometer viscoelastic jets. In this technique, the liquid jet is gently extruded by the smooth suction caused by the acceleration in the discharge orifice of a coflowing gas stream. Nozzle clogging is a rare phenomenon in flow focusing because the emitted jet is much thinner than all the passages and orifices in the device. The Weber number characterizing the jet is very small, which reduces the liquid impact velocity when it is printed onto a substrate. In this article, we report the design, build, and characterization, for the first time, of a flow focusing ejector. Using an aqueous polyacrylamide solution, we examine the dependence of the diameter and velocity of the jet upon the ejector control parameters: injected flow rate, applied pressure drop, and distance from the discharge orifice. Moreover, we also study the deposition onto a glass substrate moving at a constant speed, paying attention to the influence that this parameter has over the printing quality. Finally, we illustrate the capabilities of the flow focusing ejector by printing lines of poly(3,4-ethylenedioxythiophene):poly(styrene sulfonate) and polyvinylpyrrolidone, fluids commonly used in flexible electronics and tissue engineering, respectively.
\end{abstract}

Keywords: gaseous flow focusing, viscoelastic microjet, liquid deposition, smooth printing, bioplotting

\footnotetext{
${ }^{*}$ Corresponding Author. Tel.: +34-924-289600; fax: +34-924-289601; e-mail: ejvega@unex.es
} 


\section{Introduction}

The controlled generation of jets on the micrometer scale is of great interest in a variety of fields, such as pharmacy [1], biotechnology [2, 3], industrial engineering [4], and the food and agriculture industry [5]. Many of these applications involve the processing of polymeric viscoelastic liquids, where the interactions between the processing conditions and the fluid rheology play a fundamental role. Micrometer viscoelastic jets can be produced by applying electric fields (the so-called electrospinning) [6] or purely mechanical/hydrodynamic means. Melt spinning [7], selective withdrawal [8], co-axial atomization [9], or pneumatic melt spinning [10] fall into this last category.

Gaseous flow focusing $[11,12]$ is a well-established method to produce Newtonian sub-millimeter laminar jets, powered by a coflowing gas stream accelerated across the discharge orifice. The application of the flow focusing principle to the extrusion of viscoelastic jets has recently been studied in Ref. [13]. In that work, the fundamental differences between the gaseous focusing of Newtonian and viscoelastic liquids were examined, paying special attention to the different sources of flow instabilities. In the present work, we develop a new flow focusing ejector for extruding micrometer viscoelastic jets on a robust and stable basis. The viscoelastic flow focusing ejector deposits jets tens of microns in diameter onto a solid surface in a smooth and ordered way. The jet tapers from a much wider feeding capillary, which reduces the shear stresses applied during the injection process and avoids undesired clogging effects. Viscoelastic effects decrease the impact velocity down to values on the order of $0.1 \mathrm{~m} / \mathrm{s}$. These features confer great potential on viscoelastic flow focusing in very diverse fields. In this article, we will analyze the ejector's performance, and will show its practical capabilities by considering two potential applications: the formation of polymer films for printed electronic applications and for the fabrication of scaffolds for tissue engineering.

PEDOT:PSS (poly(3,4-ethylenedioxythiophene):poly(styrene sulfonate)) is probably the best polymer for applications in printed and flexible electronics due to its high electrical conductivity, transparency, physical and chemical stability in air, and capability to easily form films. These films can be produced with a variety of methods such as spin-coating, spin-drying, 
and different printing techniques. These printing techniques include gravure, spray [14] and screen printing, as well as imprinting and droplet-based inkjet printing [15]. In principle, inkjet printing of PEDOT-PSS constitutes a simple, flexible and fast procedure to deposit/pattern PEDOT-PSS with low cost, waste, and contamination. It has been used in the fabrication of organic solar cells [16, 17], electronic circuitry components [18], LEDs, sensors, and biosensors, among other electronic systems. One can arrange the printing conditions to connect individual dots and form lines. Also, layers connecting the lines can be produced by reducing the offset of line alignment. However, nozzle clogging frequently occurs in drop-based inkjet printing of PEDOT:PSS when attempting to increase the spatial resolution and/or velocity of printing. Additionally, ink viscosity and surface tension, together with the printhead driving waveforms, must be carefully adjusted to properly form droplets. Moreover, drop spacing and dewetting may significantly affect the electrical properties and uniformity of the film. These are some of the problems arising in inkjet printing of PEDOT:PSS that can be overcome naturally with viscoelastic flow focusing. As mentioned above, nozzle clogging is a rare phenomenon in flow focusing because the emitted jet is much thinner than all the passages and orifices of the device. The only liquid property required in the generation of viscoelastic microjets by flow focusing is a certain degree of viscoelasticity, which can be readily obtained by adding a minuscule amount of a long-chain polymer, such as polyacrylamide (PAA), to the aqueous solution. Additionally, the filament can be continuously and slowly printed onto the substrate, eliminating both drop spacing (i.e. discontinuities) and reducing the impact velocity.

Among the 3-Dimensional biofabrication techniques, one can distinguish stereolithography, Laser Induced Forward Transfer (LIFT), Laser Guidance Direct Write (LGDW) [19], drop-based inkjet printing (bioprinting) [20, 21, $22,23]$, and extrusion bioplotting (also referred to as Direct Ink Write, DIW) $[24,19,21]$. The latter technique is based on the extrusion of continuous filaments of "bioinks", stored in a syringe or a similar device and deposited in spatially controlled filaments by a robotic system. Bioinks usually consist of aqueous media, thermoreversible polymers, or polymer/hydrogel precursors combined with living cells. The typical diameter of the nozzles range from tens of microns to millimeters and are actuated by solenoid-based, piezoelectric, or pneumatic mechanisms. Bioplotting is a "structural" cell printing technique because it allows simultaneous printing of both the scaffold and the living cells (or other bioactive compounds). It is a simple and non-contact 
printing technology.

Bioplotting is not exempt from problems. When thin filaments are deposited, there is risk of clogging, and cells can be damaged by the shear stresses acting in the nozzle [25]. The impact velocities usually lie in the rage of $5-20 \mathrm{~m} / \mathrm{s}$, which may also affect the cell viability [24]. In electrohydrodynamic (EHDJ) printing, the filament is driven by the electrostatic suction produced by an applied voltage between the nozzle and the substrate. This force extrudes micrometer jets from millimeter nozzles, which considerably reduces the shear stresses over the filament extrusion. However, it is not clear whether the intense applied electric fields and the relatively large impact velocities damage the deposited cells [24]. The problems mentioned above are not present in viscoelastic flow focusing, where the jet is gently extruded by the smooth suction in the orifice due to gas acceleration. The speed of the emitted jet is decreased down to $0.1 \mathrm{~m} / \mathrm{s}$ for appropriate values of the liquid elasticity and other ejector control parameters, hence reducing cell damage upon impact. Viscoelastic flow focusing can be applied to ink solutions containing monomers (or oligomers), cross-linker molecules, and initiators for fabrication of scaffolds. This technique can also be utilized as a conformal cell printing method for printing cells and biomolecules on top of thin layers of prefabricated scaffolding. We will illustrate the capabilities of the flow focusing ejector by printing lines of polyvinylpyrrolidone (PVP), a polymer frequently used in tissue engineering.

It must be noted that viscoelastic flow focusing has the potential to be applicable in other fields as well. For instance, it is well-known that the continuous jet impingement of a liquid onto a surface can be used as a highcapacity cooling method. The addition of small amounts (on the order of $100 \mathrm{ppm}$ ) of polymers to water-based jets leads to a noticeable increase of the cooling rate as compared to that of pure water [26]. The impingement water-based suspension of polymers can also be used to reduce the friction in rails. In this case, the liquid is usually applied onto the rail top surface with sprayers mounted on maintenance vehicles [27]. Viscoelastic flow focusing can constitute an advantageous technology in these fields too.

\section{The viscoelastic flow focusing ejector}

Figure 1-left shows a photograph of the flow focusing ejector designed in this work. A feeding capillary is located inside a glass tube with a converging nozzle formed at its end [28]. We make use of a spring (spiral-like metal 
structural) to alight the capillary in the center (inside) of the glass tube. The viscoelastic liquid is injected at a constant flow rate $Q$ through the capillary. An air stream flows through the tube and exits at the nozzle driven by an applied pressure drop $\Delta p$. Due to the constriction, the air accelerates near the nozzle in a region the size of the nozzle diameter [13]. The depression produced by that acceleration sucks the liquid, tapering it from the feeding capillary to a very thin jet. This suction produces an axial stress in the viscoelastic liquid which is transmitted upstream hardening the ejected filament. The filament is expelled through the nozzle orifice with a diameter $d_{j}$ which decreases downstream due to the drag force exerted by the air stream. The polymer molecules are stretched and arranged inside the jet in such a way that the effective liquid viscosity soars. The macromolecule relaxation time is much longer than the fluid particle residence time, and therefore the viscosity does not recover to the relaxed state value during ejection. As a consequence, the jet can travel distances hundreds of times its diameter without breaking up. Figure 1-right shows a sketch of the flow focusing ejector with the main characteristic lengths. The inner diameter of both the feeding capillary and glass tube are $D_{c}$ and $D_{t}$, respectively. The nozzle orifice has diameter $D_{o}$, and is separated by a distance $H$ from the end of the capillary.
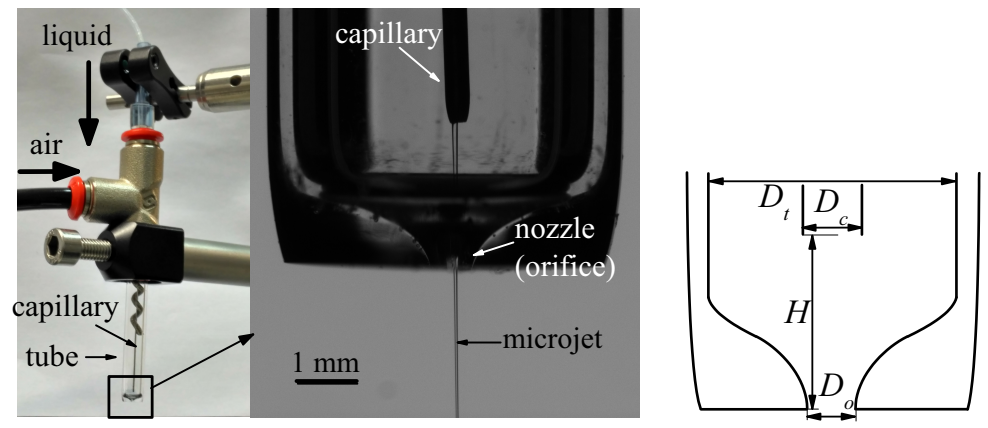

Figure 1: Photograph of the flow focusing ejector (left) and sketch (right) showing the main characteristic lengths: $D_{c}=200 \mu \mathrm{m}, D_{t}=4 \mathrm{~mm}$ and $D_{o}=375 \mu \mathrm{m}$.

The inner diameter of the glass tube, $D_{t}$, plays no significant role in the liquid ejection as long as it takes values much larger than the rest of characteristic lengths. The inner diameter of the capillary, $D_{c}$, does not significantly affect the ejector performance if the jet is unpinned from the 
capillary edge (see explanation below). The diameter $D_{o}$ of the nozzle orifice considerably affects the jetting stability. The pressure gradient arising in the suction region scales as $\Delta p / D_{o}$, and therefore it decreases as $D_{o}$ increases. If the pressure gradient is too small (the orifice is too big), the air does not sufficiently suck the liquid and the jet does not form. On the contrary, if the pressure gradient is too large (the orifice is too small), the high axial stress in the viscoelastic liquid prevents the transition from the Poiseuille-like flow in the capillary to the plug flow in the jet. Based on these considerations, we selected the values $D_{c}=200 \mu \mathrm{m}, D_{t}=4 \mathrm{~mm}$ and $D_{o}=375 \mu \mathrm{m}$,

Ponce-Torres et al. [13] analyzed the effect of the feeding capillary-toorifice distance $H$ on the steady jetting stability. For fixed values of $D_{o}, Q$ and $\Delta p$, there is an interval of $H$ for which the jet forms anchored to the edge of the capillary end. If the distance $H$ takes too large values, the viscoelastic axial stress originating in the nozzle orifice cannot hold the liquid filament formed between the feeding capillary and that orifice. On the contrary, too small values of $H$ lead to very large axial stresses, which also destabilizes the ejection. In this case, steady jetting can still be obtained but the viscoelastic axial stress unpins the jet from the capillary edge (the so-called "pull-out" effect). It must be noted that the pull-out effect does not constitute any obstacle to produce steady filaments.

In Ref. [13], flow focusing was produced by suctioning air from the ambient through an orifice. The feeding capillary was located in front of that orifice in the open air, and the value of $H$ could be varied and selected during the liquid ejection to ensure perfect steady jetting. The ejector developed here works with a fixed capillary-to-orifice distance, and therefore the viscoelastic jet must form autonomously, i.e., without requiring any manipulation of the user apart from switching on the pumps of liquid and air. We have verified that there is a relatively narrow range of $H$ for that to occur. Within that range, autonomous (self-sustained) steady jetting with the pull-out effect is obtained for flow rates and pressure drops similar to those in Ref. [13]. Both the upper and lower limits of this interval increase with the polymer relaxation time. Based on these considerations, we selected the values $H=2.5$ and $0.9 \mathrm{~mm}$ for the solutions containing PAA and PVP, respectively.

Once these geometrical values are fixed, the jet diameter $d_{j}$ and velocity $v_{j}=4 Q /\left(\pi d_{j}^{2}\right)$ are functions of the flow rate $Q$ and pressure drop $\Delta p$. Bioplotting requires small diameters and velocities, which demands sufficiently low flow rates and applied pressure drops. There is an additional reason for 
reducing the applied pressure drop. It is well known that whipping instability can be triggered by the difference between the jet and surrounding air velocities [28]. In flow focusing, this difference is caused by the high speed of the coflowing air current near the discharge orifice and the velocity of the jet when moving in the relatively quiescent surrounding air far away from the ejector. In both cases, the velocity difference decreases as the pressure drop decreases, which prevents the jet from whipping. We have found that the ranges $1 \mathrm{ml} / \mathrm{h} \leq Q \leq 5 \mathrm{ml} / \mathrm{h}$ and $25 \mathrm{mbar} \leq \Delta p \leq 95 \mathrm{mbar}$ are adequate for the applications shown in Sec. 3. The latter range approximately corresponds to gas speeds between $64 \mathrm{~m} / \mathrm{s}$ and $125 \mathrm{~m} / \mathrm{s}$. For smaller flow rates, gobbling [29] takes place at the end of the feeding capillary, and the steady jetting regime cannot be established, while for smaller pressure drops the suction effect is not intense enough for the ejection to occur.

In this work, experiments were conducted to analyze the dependence of the jet diameter $d_{j}$ and velocity $v_{j}$ as a function of the flow rate $Q$ for different applied pressure drops $\Delta p$ within the ranges mentioned above. The liquid was injected with a syringe pump (PHD 4400, Harvard Apparatus), while the gaseous current was provided by a pneumatic circuit whose upstream stagnation pressure was fixed and controlled with a high-precision pressure regulating valve. The ejector was oriented with a dual-axis goniometer. Digital images of $1280 \times 960$ pixels were acquired at 30 frames per second with back-side illumination using a CCD camera (AVT STRINGAY F-125B) equipped with optical lenses (an OpTEM ZOOM 70XL set of lenses with variable magnification from $0.75 \times$ to $5.25 \times$ ) providing a frame covering an area of about $96.78 \times 72.58 \mu \mathrm{m}$. The magnification obtained was approximately $76 \mathrm{~nm} /$ pixel. The camera could be displaced both horizontally and vertically using a triaxial translation stage. The working fluid consisted of an aqueous solution of PAA with a concentration of $1000 \mathrm{ppm}$. The density and surface tension of this polymeric solution are $\rho \simeq 997 \mathrm{~kg} / \mathrm{m}^{3}$ and $\sigma \simeq 72 \mathrm{mN} / \mathrm{m}$, respectively. The zero shear viscosity and relaxation time are $\eta_{0} \simeq 10 \mathrm{~Pa} \mathrm{~s}$ and $\lambda \simeq 140 \mathrm{~ms}$, respectively [30]. It must be noted that the jet's diameter and velocity may slightly change due to small variations of the relaxation time when a new solution is fabricated.

Figure 2 shows the results for jets that exhibited small lateral oscillations (in all cases the amplitude were smaller than four times the jet's diameter). As expected, the jet's diameter decreases as the flow rate decreases. Both the pressure drop and the viscous stresses exerted by the high-speed coflowing air stream transfer energy to the viscoelastic jet. The work done by the gas 
viscous stresses is proportional to the free surface area. Therefore, the energy transferred per unit volume scales as $d_{j}^{-1}$. This explains why $v_{j}$ increases as $Q$ (and therefore $d_{j}$ ) decreases for a fixed applied pressure drop. Moroever, it explains why $v_{j}$ increases with the distance $Z$ from the discharge orifice. In addition, the diameter (velocity) decreases (increases) as the applied pressure drop increases for a fixed flow rate. The jet's velocities are much smaller than those in the Newtonian flow focusing technique [11, 12], $v_{j} \simeq(2 \Delta p / \rho)^{1 / 2}$. This is due to the fact that most of the injected energy is converted into interfacial and elastic energies. This can be regarded as an advantage over the Newtonian case as the jet's velocity must be sufficiently small for printing gently onto a substrate.
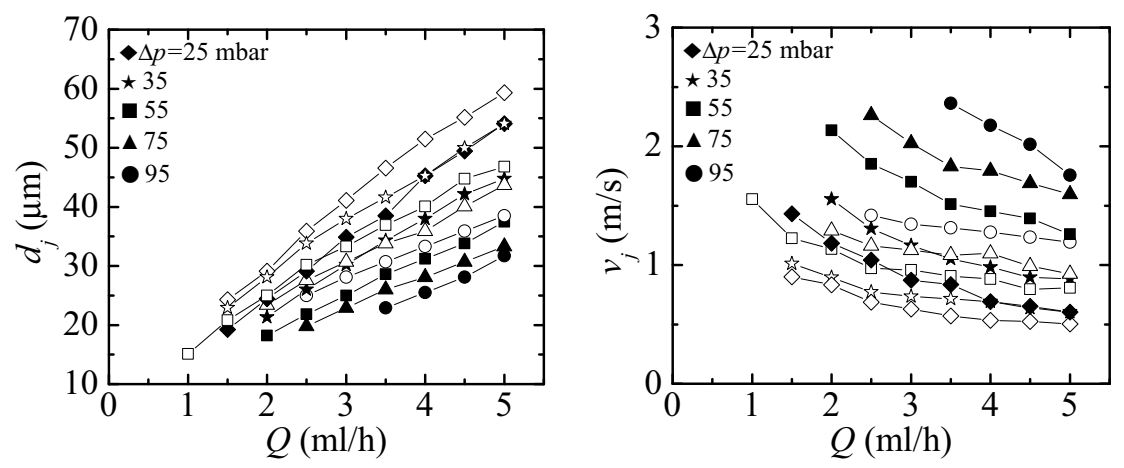

Figure 2: Jet diameter $d_{j}$ (left) and velocity $v_{j}$ (right) as a function of the flow rate $Q$ for several applied pressures $\Delta p$. The open and solid symbols correspond to the distance from the ejector $Z=1$ and $2 \mathrm{~mm}$, respectively. The results were obtained for $H=2.5$ $\mathrm{mm}$. The liquid was a PAA aqueous solution $1000 \mathrm{ppm}$ in concentration.

\section{Printing viscoelastic liquids}

Figure 3 shows a picture of the experimental setup used in the printing experiments, as well as two sketches of the printing process and the deposited rivulet. Images of the deposition process were acquired with a high-speed CMOS camera (Photron, FAstcam SA5) coupled to a microscope (an OPTEM ZOOM 70XL set of lenses with variable magnification from $0.75 \times$ to $5.25 \times$ ) from the lateral side and from below. In the latter case, the liquid deposition was illuminated by an optical fiber from above, and the image reflected by a mirror was acquired by the camera. The substrate consisted of 
a glass slide (SELECT Micro Slides) with a roughness in the order of a few nanometers. The glass slide was carefully cleaned with isopropanol and dried before use in order to prevent contamination. Experiments were conducted at atmospheric pressure and room temperature $20 \pm 2{ }^{\circ} \mathrm{C}$. The ejector was located at a distance $Z$ above the flat surface (substrate), which was displaced horizontally at a constant velocity $v_{s}$. A viscoelastic jet of diameter $d_{j}$ was emitted with a velocity $v_{j}$ by injecting liquid at a flow rate $Q$ and pressure drop $\Delta p$. The jet's diameter (velocity) was slightly smaller (larger) than that measured without liquid deposition (Fig. 2) due to the traction exerted by the solid surface (the fiber spinning effect for $v_{s}>v_{j}[7]$ ). In addition, the presence of the substrate reduces considerably the lateral vibration of the liquid thread formed between the ejector and that substrate. For this reason, whipping does not constitute an important obstacle for reproducible printing within the selected ranges of flow rates and applied pressure drops. For appropriate values of $v_{s}$, a rivulet of width $w$ and height $h$ formed after the liquid spread over the glass surface. In all the cases, the rivulet remained stable during the time period over which images were acquired. The rivulet subsequently broke up into droplets due to the evaporation of the solvent.

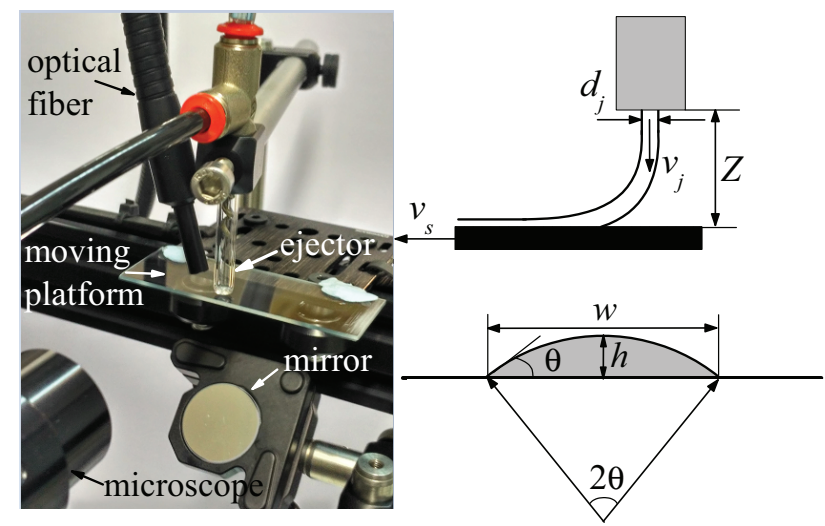

Figure 3: Photograph of the experimental setup used in the printing experiments, and sketches of the printing process and the deposited rivulet.

\subsection{Printing an PAA aqueous solution at a concentration of 1000 ppm}

The viscoelastic flow focusing ejector described above was used to print strips of a PAA aqueous solution $1000 \mathrm{ppm}$ in concentration onto a glass 
surface. A viscoelastic jet of diameter $d_{j} \simeq 60 \mu \mathrm{m}$ is emitted with a velocity $v_{j} \simeq 0.5 \mathrm{~m} / \mathrm{s}$ by injecting liquid with $Q=5 \mathrm{ml} / \mathrm{h}$ and $\Delta p=35$ mbar. Figure 4 shows the lateral and top views of the deposition process for $Z=1 \mathrm{~mm}$ and values of the surface velocity within the interval $0.2 \mathrm{~m} / \mathrm{s} \leq v_{s} \leq 0.95$ $\mathrm{m} / \mathrm{s}$. For $v_{s}<0.2 \mathrm{~m} / \mathrm{s}$, both the rivulet width and non-uniformity degree sharply increase, while for $v_{s}>0.95 \mathrm{~m} / \mathrm{s}$ the viscoelastic jet touches the ejector and the deposition is intermittently interrupted. The rivulet nonuniformity may be caused by fluctuations in the ejection process, and more likely by instabilities at the (dynamic) contact lines during impact and spread of the liquid over the solid surface [31]. As can be observed, the flow focusing ejector is capable of printing submillimeter straight strips with a high degree of uniformity. The offset between the printing point and the actual contact point is similar to those found in Refs. [32, 33]. As shown in Ref. [34], this distance increases as the speed of the substrate increases.
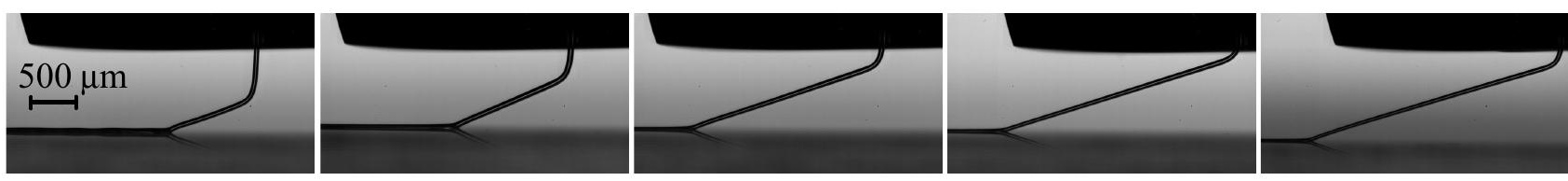

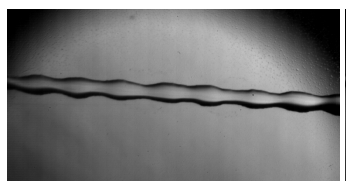

$v_{\mathrm{s}}=0.22 \mathrm{~m} / \mathrm{s}$

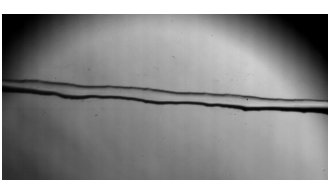

$0.44 \mathrm{~m} / \mathrm{s}$

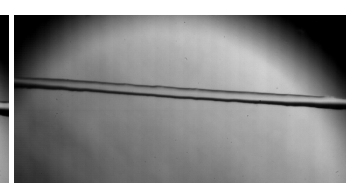

$0.65 \mathrm{~m} / \mathrm{s}$

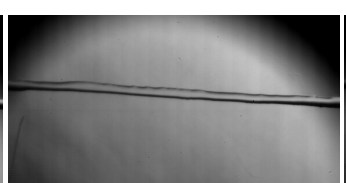

$0.85 \mathrm{~m} / \mathrm{s}$

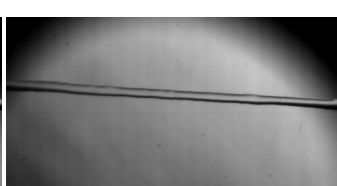

$0.95 \mathrm{~m} / \mathrm{s}$

Figure 4: Lateral (upper images) and bottom (lower images) views of the deposition process for different values of the surface velocity $v_{s}$. From left to right, the images correspond to $v_{s}=0.22,0.44,0.65,0.85$ and $0.95 \mathrm{~m} / \mathrm{s}$. The results were obtained for $H=2.5 \mathrm{~mm}, Q=5 \mathrm{ml} / \mathrm{h}, \Delta p=35 \mathrm{mbar}$, and $Z=1 \mathrm{~mm}$. The liquid was a PAA aqueous solution $1000 \mathrm{ppm}$ in concentration.

The rivulet width distribution $w(x)$ along the rivulet axis was measured by processing the bottom views with a subpixel resolution image analysis technique [35]. Figure 5-left shows the average values $\bar{w}$ as a function of the surface velocity $v_{s}$. The error bars correspond to the standard deviations of the distributions $w(x)$, and indicate the degree of the rivulet uniformity. Their relatively small values indicate that the resulting footprint is fairly uniform. Due to the smallness of the rivulets, the Bond number $B=\rho g h^{2} / \sigma$ takes very low values $\left(B \sim 10^{-4}\right)$, and therefore the contour deformation due 
to the gravitational force can be neglected. If one assumes that the rivulet's cross section corresponds to a circular segment, the conservation of liquid volume leads to the equation

$$
k \equiv \frac{2 \bar{\theta}-\sin (2 \bar{\theta})}{\sin ^{2} \bar{\theta}}=2 \pi\left(\frac{d_{j}}{\bar{w}}\right)^{2} \frac{v_{j}}{v_{s}},
$$

where $\bar{\theta}$ is the (average) contact angle. Figure 5-right shows the values of $k$ given by Eq. (1) as a function of the surface velocity $v_{s}$. As expected, this parameter takes an almost constant value. The average value of $k$ corresponds to $\bar{\theta} \simeq 48^{\circ}$. This value is consistent with our contact angle measurement for the same system using theoretical image fitting analysis (TIFA) [36]. The average rivulet height $\bar{h}$ can also be estimated as $\bar{h}=\bar{w}(1-\cos \bar{\theta}) /(2 \sin \bar{\theta})$ (Fig. 3). Figure 5-left shows the average heights of the rivulet estimated from the above formula.
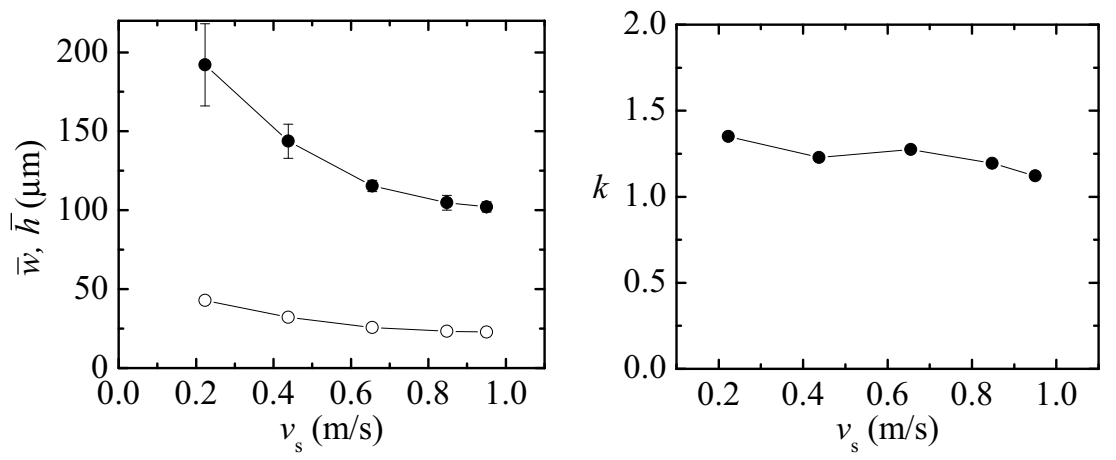

Figure 5: (Left) Average values $\bar{w}$ (solid symbols) and $\bar{h}$ (open symbols) as a function of the surface velocity $v_{s}$. The error bars correspond to the standard deviations of the distributions $w(x)$. (Right) Values of $k$ as a function of the surface velocity $v_{s}$. These results were obtained for $H=2.5 \mathrm{~mm}, Q=5 \mathrm{ml} / \mathrm{h}, \Delta p=35 \mathrm{mbar}$, and $Z=1 \mathrm{~mm}$. The liquid was a PAA aqueous solution $1000 \mathrm{ppm}$ in concentration.

Figure 6 shows the influence of the distance $Z$ between the ejector and the solid surface. For $Z=1 \mathrm{~mm}$, the printing process is quasi-steady. For $Z=2$ and $3 \mathrm{~mm}$, the jet 'bounces' periodically on the solid surface, which produces the vertical oscillation of the height at which the jet bends [34]. This oscillation produces the accumulation of liquid at equally spaced rivulet sections. However, and due to the subsequent redistribution of liquid along 
the rivulet, the resulting footprint is fairly uniform. Therefore, the printing quality does not decrease significantly as the distance $Z$ from the ejector increases. This is beneficial for printing applications that demand a minimum distance between the printhead and the substrate. As explained above, there is more room for the coflowing air stream to drag the liquid jet as the ejectorto-surface distance $Z$ increases. This energy transfer, from the gas to the liquid, results in an increase of the jet speed, and therefore in a reduction of its diameter (Fig. 2). This explains why the jet in the central and lower images of Fig. 6 is significantly thinner than in the upper ones.
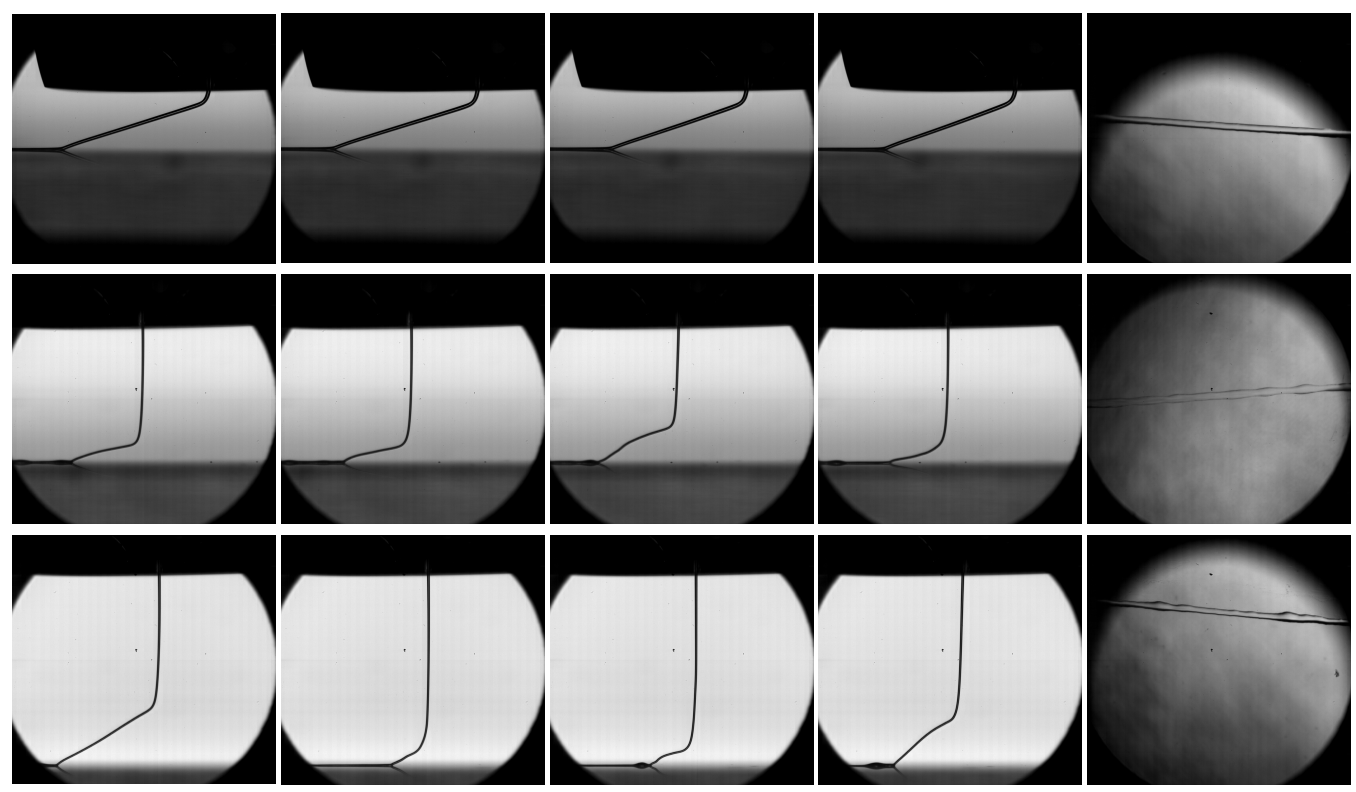

Figure 6: Lateral and bottom views of the deposition process for $Z=1$ (upper images), 2 (central images), and $3 \mathrm{~mm}$ (lower images). The lateral view images correspond to different instants of the printing process. The results were obtained for $H=2.5 \mathrm{~mm}$, $Q=5 \mathrm{ml} / \mathrm{h}, \Delta p=35 \mathrm{mbar}$, and $v_{s}=0.65 \mathrm{~m} / \mathrm{s}$. The liquid was a PAA aqueous solution $1000 \mathrm{ppm}$ in concentration. The jet's diameter in the upper, central, and lower images was 66,52 , and $47 \mu \mathrm{m}$, respectively.

\subsection{Applications}

As mentioned in the Introduction, PEDOT:PSS is probably the best conducting polymer for applications in electronics. Printing lines and layers of 
this liquid in a controlled manner is of great interest to fabricate varied electronic systems. PEDOT:PSS aqueous solutions exhibit a near-Newtonian character with a density and viscosity similar to those of water. In fact, the low elasticity of this solution prevents its printing with the flow focusing ejector. In order to enhance the viscoelastic behavior of the PEDOT:PSS solution, a small amount of PAA can be dissolved in that liquid. When PAA is added at $0.2 \mathrm{wt} \%$, the relaxation time increases from around $100 \mu \mathrm{s}$ up to around $200 \mathrm{~ms}$. Figure 7 shows the printing of an aqueous solution of PEDOT:PSS at a concentration around $3 \mathrm{wt} \%$ to which $0.2 \mathrm{wt} \%$ of PAA has been added. The density and surface tension of this polymer solution are $\rho \simeq 990 \mathrm{~kg} / \mathrm{m}^{3}$ and $\sigma \simeq 46 \mathrm{mN} / \mathrm{m}$, while the zero shear viscosity is $\eta_{0} \simeq 100$ $\mathrm{Pa}$ s. The figure also shows the footprint obtained after water evaporates. A continuous straight strip around $90 \mu \mathrm{m}$ in width is printed out with our flow focusing ejector. We verified that the electric conductivity of the printed lines was similar to the value expected in the absence of the PAA additive.

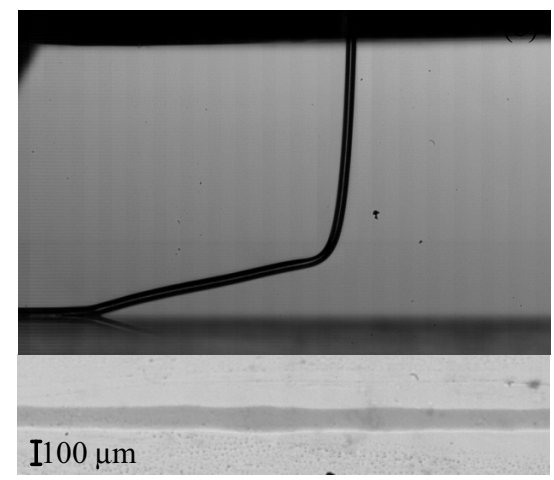

Figure 7: Lateral view of the PEDOT:PSS deposition process (upper image) and the footprint that remains indefinitely after deposition (lower image). The results were obtained for $H=2.5 \mathrm{~mm}, Q=5 \mathrm{ml} / \mathrm{h}, \Delta p=40 \mathrm{mbar}, Z=1.5 \mathrm{~mm}$, and $v_{s}=0.65 \mathrm{~m} / \mathrm{s}$. The liquid was an aqueous solution of PEDOT:PSS at a concentration of around $3 \mathrm{wt} \%$ to which 0.2 wt $\%$ of PAA was added.

Ink solutions containing monomers (or oligomers), cross-linker molecules, and initiators can be printed with flow focusing for the fabrication of scaffolds. Flow focusing can also be used to print cells and biomolecules on top of thin layers of prefabricated scaffolding. The present technique reduces damaging effects on those cells and biomolecules by reducing both the shear stresses acting in the liquid and the impact velocities. We illustrate this possibility 
by extruding filaments of an aqueous solution of PVP at a concentration of $10 \mathrm{wt} \%$, and depositing them onto a glass surface. The density and surface tension of this polymer solution are $\rho \simeq 982 \mathrm{~kg} / \mathrm{m}^{3}$ and $\sigma \simeq 48 \mathrm{mN} / \mathrm{m}$, while the zero shear viscosity and relaxation times are $\eta_{0} \simeq 0.1 \mathrm{~Pa} \mathrm{~s}$ and $\lambda \simeq 10 \mathrm{~ms}$, respectively. This liquid has less elasticity than the previous solutions, which yields higher jet velocities. In addition, the orifice-capillary distance must be reduced to get autonomous steady jetting. The upper image of Fig. 8 shows the lateral view of the PVP deposition process. The process was repeated 60 times leaving water to evaporate between two consecutive depositions. In this way, a "wall" of PVP around $50 \mu \mathrm{m}$ in height and $90 \mu \mathrm{m}$ in width was formed. The lower image shows the footprint after the first deposition.

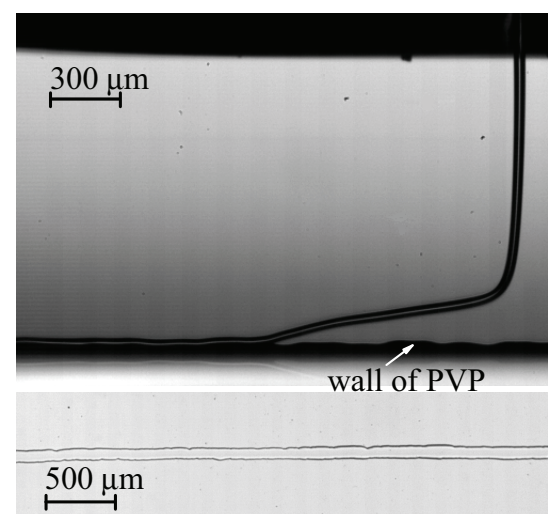

Figure 8: Lateral view of the PVP deposition process (upper image) and the footprint after the first deposition (lower image). The process was repeated 60 times to build a wall of PVP around $50 \mu \mathrm{m}$ in height and $90 \mu \mathrm{m}$ in width. The results were obtained for $H=0.9 \mathrm{~mm}, Q=4 \mathrm{ml} / \mathrm{h}, \Delta p=35 \mathrm{mbar}, Z=1 \mathrm{~mm}$, and $v_{s}=0.95 \mathrm{~m} / \mathrm{s}$. The liquid was an aqueous solution of PVP at a concentration of $10 \mathrm{wt} \%$.

\section{Conclusions}

The gaseous flow focusing principle as applied to viscoelastic liquids allows for the smooth extrusion of micrometer jets thanks to the suction effect caused by the coflowing air stream when crossing the discharge orifice. The axial elastic stress originated in that region propagates both upstream and downstream hardening the liquid filament and suppressing the capillary instability. The emitted jet is much thinner than all device passages and 
orifices, which prevents the ejector from clogging. Elasticity becomes an important sink of energy. As a result, the jet kinetic energy is much smaller than the interfacial one, which considerably reduces the impact velocity during the liquid deposition. These features make the gaseous flow focusing principle an excellent candidate for micrometer printing in very varied fields ranging from electronics to tissue engineering.

In this work, we have designed and built for the first time an ejector based on the gaseous flow focusing principle to extrude viscoelastic jets for printing applications. The proposed design shows to be robust and easy to use because the jet forms in a reproducible manner and autonomously, without requiring any manipulation from the user apart from switching on the pumps of liquid and air. We have characterized the flow focusing ejector by measuring the diameter and speed of a PAA-aqueous-solution jet for different values of the major control parameters: the liquid flow rate, the air pressure drop, and the distance from the discharge orifice. The air stream does not trigger the whipping instability within the range of applied pressure drops. As expected, the jet can be gently deposited onto a flat surface if the jet and surface velocities take similar values. Straight rivulets with an almost constant width are formed after deposition. While the optimum ejector-tosurface distance was around $1 \mathrm{~mm}$, good results were also obtained for larger distances.

In order to illustrate the potential applications of the technique presented here, we printed films of PEDOT:PSS and PVP, which are frequently used in electronics and tissue engineering, respectively. In the PEDOT:PSS case, PAA was added at a very small concentration to enhance the viscoelastic nature of the polymeric solution. Continuous, straight, and uniform strips around $90 \mu \mathrm{m}$ in width were succesfully printed with our flow focusing ejector. A wall of PVP around $50 \mu \mathrm{m}$ in height and $90 \mu \mathrm{m}$ in width was built by repeating the deposition process. The spatial resolution of this technique is expected to increase when printing onto hydrophobic substrates.

It is well known that the shear stresses arising in bioprinting during feeding through thin capillaries (nozzles) may damage living cells [25]. Consider an application where a liquid thread of around $50 \mu \mathrm{m}$ in diameter is ejected at a flow rate $Q=3 \mathrm{ml} / \mathrm{h}$. Typically, the inner nozzle diameter for such an application is about $30 \mu \mathrm{m}$ [37], which leads to shear rates of the order of $10^{5} \mathrm{~s}^{-1}$. Assume that, for instance, the liquid viscosity is of the order of $1 \mathrm{~Pa} \mathrm{~s}$. In this case, the viscous shear stress takes values of the order of $10^{5} \mathrm{~Pa}$, which can reduce the percentage cell viability by $30 \%$ [25]. Now, 
suppose that we replace the nozzle with the viscoelastic flow focusing ejector. In order to produce the focusing effect, a small amount of PAA can be added without significantly changing the mixture shear viscosity due to the shear thinning behavior of PAA. The inner diameter of the feeding capillary becomes around $200 \mu \mathrm{m}$, which reduces the shear stresses by two orders or magnitude. This reduction almost eliminates the possibility of cell damage $[25,38]$.

Partial support from the Ministry of Science and Education and Gobierno de Extremadura (Spain) through Grants Nos. DPI2016-78887 and GR10047, respectively, is gratefully acknowledged. AACP acknowledges the support from the Royal Society through a University Research Fellowship. We thank J.-C. Tan for helpful discussions and suggestions regarding the test fluids.

[1] A. M. Gañán-Calvo, J. M. Montanero, L. Martín-Banderas, M. FloresMosquera, Building functional materials for health care and pharmacy from microfluidic principles and Flow Focusing, Adv. Drug Delivery Rev. 65 (2013) 1447-1469.

[2] D. P. DePonte, U. Weierstall, K. Schmidt, J. Warner, D. Starodub, J. C. H. Spence, R. B. Doak, Gas dynamic virtual nozzle for generation of microscopic droplet streams, J. Phys. D: Appl. Phys. 41 (2008) 195505.

[3] H. N. Chapman et al., Femtosecond x-ray protein nanocrystallography, Nature 470 (2011) 73-79.

[4] M. Orme, Q. Liu, R. Smith, Molten aluminum micro-droplet formation and deposition for advanced manufacturing applications, Aluminum Transactions 3 (2000) 95-103.

[5] I. Cohen, H. Li, J. L. Hougland, M. Mrksich, S. R. Nagel, Using selective withdrawal to coat microparticles, Science 292 (2001) 265-267.

[6] Z.-M. Huanga, Y.-Z. Zhang, M. Kotaki, S. Ramakrishna, A review on polymer nanofibers by electrospinning and their applications in nanocomposites, Compos. Sci. Technol. 63 (2003) 2223-2253. 
[7] M. M. Denn, Continuous drawing of liquids to form fibers, Ann. Rev. Fluid Mech. 12 (1980) 365-387.

[8] D. Zhoua, J. J. Feng, Selective withdrawal of polymer solutions: Experiments, J. Non-Newtonian Fluid Mech. 165 (2010) 829-838.

[9] A. Mansour, N. Chigier, Air-blast atomization of non-newtonian liquids, J. Non-Newtonian Fluid Mech. 58 (1995) 161-194.

[10] A. Zachara, Z. Lewandowski, Mathematical modelling of pneumatic melt spinning of isotactic polypropylene. Part I. Modelling of the air jet dynamics, Fibres and Textiles in Eastern Europe 16 (2008) 17-23.

[11] A. M. Gañán-Calvo, Generation of steady liquid microthreads and micron-sized monodisperse sprays in gas streams, Phys. Rev. Lett. 80 (1998) 285-288.

[12] A. M. Gañán-Calvo, J. M. Montanero, Revision of capillary cone-jet physics: Electrospray and flow focusing, Phys. Rev. E 79 (2009) 066305.

[13] A. Ponce-Torres, J. M. Montanero, E. J. Vega, A. M. Gañán-Calvo, The production of viscoelastic capillary jets with gaseous flow focusing, J. Non-Newtonian Fluid Mech. 229 (2016) 8-15.

[14] K. X. Steirer, J. J. Berry, M. O. Reese, M. F. van Hest, A. Miedaner, M. W. Liberatore, R. Collins, D. S. Ginley, Ultrasonically sprayed and inkjet printed thin film electrodes for organic solar cells, Thin Solid Films 517 (2009) 2781-2786.

[15] S. Jung, A. Sou, K. Banger, D.-H. Ko, P. C. Y. Chow, C. R. McNeill, H. Sirringhaus, All-inkjet-printed, all-air-processed solar cells, Adv. Energy Mater. 4 (2014) 1400432.

[16] A. Singh, M. Katiyarab, A. Garg, Understanding the formation of PEDOT:PSS films by ink-jet printing for organic solar cell applications, RSC Adv. 5 (2015) 78677-78685.

[17] K. Sun, S. Zhang, P. Li, Y. Xia, X. Zhang, D. Du, F. H. Isikgor, J. Ouyang, Review on application of PEDOTs and PEDOT:PSS in energy conversion and storage devices, J. Mater. Sci.: Mater. Electron. 26 (2015) 4438-4462. 
[18] Y. Guo, M. T. Otley, M. Li, X. Zhang, S. K. Sinha, G. M. Treich, G. A. Sotzing, PEDOT:PSS "wires" printed on textile for wearable electronics, ACS Appl. Mater. Interfaces 8 (2016) 26998-27005.

[19] S. Wust, R. Muller, S. Hofmann, Controlled positioning of cells in biomaterials-approaches towards 3D tissue printing, J. Funct. Biomater 2 (2011) 119-154.

[20] S. Jung, S. D. Hoath, I. M. Hutchings, The role of viscoelasticity in drop impact and spreading for inkjet printing of polymer solution on a wettable surface, Microfluid Nanofluid 14 (2013) 163-169.

[21] P. Bajaj, R. M. Schweller, A. Khademhosseini, J. L. West, R. Bashir, 3d biofabrication strategies for tissue engineering and regenerative medicine, Annu. Rev. Biomed. Eng. 16 (2014) 247-276.

[22] A. Faulkner-Jones, C. Fyfe, D.-J. Cornelissen, J. Gardner, J. King, A. Courtney, W. Shu, Bioprinting of human pluripotent stem cells and their directed differentiation into hepatocyte-like cells for the generation of minilivers in 3d, Biofabrication 7 (2015) 044102.

[23] H. Gudapati, M. Dey, I. Ozbolat, A comprehensive review on dropletbased bioprinting: Past, present and future, Biomaterials 102 (2016) $20-42$.

[24] B. R. Ringeisen, C. M. Othon, J. A. Barron, D. Young, B. J. Spargo, Jet-based methods to print living cells, Biotechnol. J. 1 (2006) 930-948.

[25] K. Nair, M. Gandhi, S. Khalil, K. C. Yan, M. Marcolongo, K. Barbee, W. Sun, Characterization of cell viability during bioprinting processes, Biotechnol. J. 4 (2009) 1168-1177.

[26] I. Sarkar, D. K. Behera, J. M. Jha, S. K. Pal, S. Chakraborty, Effect of polymer additive on the cooling rate of a hot steel plate by using water jet, Exp. Therm. Fluid Sci. 70 (2016) 105-114.

[27] B. Keshavarz, S. I. Green, D. T. Eadie, Elastic liquid jet impaction on a high-speed moving surface, AIChE J. 58 (2012) 3568-3577. 
[28] A. J. Acero, C. Ferrera, J. M. Montanero, A. M. Gañán-Calvo, Focusing liquid microjets with nozzles, J. Micromech. Microeng. 22 (2012) 065011.

[29] C. Clasen, J. Bico, V. M. Entov, G. H. McKinley, "Gobbling drops": the jetting-dripping transition in flows of polymer solutions, J. Fluid Mech 636 (2009) 5-40.

[30] P. C. Sousa, E. J. Vega, R. G. Sousa, J. M. Montanero, M. A. Alves, Measurement of relaxation times in extensional flow of weakly viscoelastic polymer solutions, Rheol. Acta 56 (2017) 11-20.

[31] M. A. Spaid, G. M. Homsy, Stability of newtonian and viscoelastic dynamic contact lines, Phys. Fluids 8 (1996) 460-478.

[32] S. Chiu-Webster, J. R. Lister, The fall of a viscous thread onto a moving surface: a "fluid-mechanical sewing machine", J. Fluid Mech. 569 (2006) $89-111$.

[33] P.-T. Brun, B. Audoly, N. M. Ribe, T. S. Eaves, J. R. Lister, Liquid ropes: A geometrical model for thin viscous jet instabilities, Phys. Rev. Lett. 114 (2015) 174501.

[34] See supplemental material at [url will be inserted by publisher] for four videos that show lateral views of the deposition process for $z=1,2$, and $3 \mathrm{~mm}$, corresponding to the experiments shown in Fig. 6, and the effect of increasing the velocity of the substrate while keeping the speed of the jet constant.

[35] E. J. Vega, J. M. Montanero, C. Ferrera, Exploring the precision of backlight optical imaging in microfluidics close to the diffraction limit, Measurement 44 (2011) 1300-1311.

[36] M. G. Cabezas, A. Bateni, J. M. Montanero, A. W. Neumann, Determination of surface tension and contact angle from the shapes of axisymmetric fluid interfaces without use of apex coordinates, Langmuir 22 (2006) 10053-10060.

[37] C. M. Smith, A. L. Stone, R. L. Parkhill, R. L. Stewart, M. W. Simpkins, A. M. Kachurin, W. L. Warren, S. K. Williams, Three-dimensional 
bioassembly tool for generating viable tissue-engineered constructs, Tissue Eng. 10 (2004) 1566-1576.

[38] M. G. Li, X. Y. Tian, X. Chen, Temperature effect on the shear-induced cell damage in biofabrication, Artif. Organs 35 (2011) 741-746. 